\title{
Antioxidant Activity and Cardioprotective Effect of a Nonalcoholic Extract of Vaccinium meridionale Swartz during Ischemia-Reperfusion in Rats
}

\author{
Yasmin E. Lopera, ${ }^{1}$ Juliana Fantinelli, ${ }^{2}$ Luisa F. González Arbeláez, ${ }^{2}$ \\ Benjamín Rojano, ${ }^{1}$ José Luis Ríos, ${ }^{3}$ Guillermo Schinella, ${ }^{4}$ and Susana Mosca ${ }^{2}$ \\ ${ }^{1}$ Laboratorio de Ciencia de Alimentos, Universidad Nacional de Colombia, Sede Medellín, Medellín, Colombia \\ ${ }^{2}$ CONICET, Centro de Investigaciones Cardiovasculares, Facultad de Ciencias Médicas, \\ Universidad Nacional de La Plata, La Plata, Argentina \\ ${ }^{3}$ Departament de Farmacologia, Facultat de Farmàcia, Universitat de València, Burjassot, 46100 Valencia, Spain \\ ${ }^{4}$ Cátedra de Farmacología Básica, Facultad de Ciencias Médicas, \\ Universidad Nacional de La Plata and CIC Provincia de Buenos Aires, La Plata, Argentina
}

Correspondence should be addressed to Susana Mosca; smosca@atlas.med.unlp.edu.ar

Received 30 May 2012; Revised 13 December 2012; Accepted 21 December 2012

Academic Editor: Adair Roberto Soares Santos

Copyright (C) 2013 Yasmin E. Lopera et al. This is an open access article distributed under the Creative Commons Attribution License, which permits unrestricted use, distribution, and reproduction in any medium, provided the original work is properly cited.

\begin{abstract}
Our objective was to assess the antioxidant properties and the effects against the reperfusion injury of a nonalcoholic extract obtained by fermentation from the Colombian blueberry, mortiño (Vaccinium meridionale Swartz, Ericaceae). Antioxidant properties were assessed by in vitro systems. To examine the postischemic myocardial function, isolated rat hearts were treated $10 \mathrm{~min}$ before ischemia and during the first $10 \mathrm{~min}$ of reperfusion with the extract. To analyze the participation of nitric oxide (NO), other experiments were performed in the presence of nitric oxide synthase (NOS) inhibition with $\mathrm{N}^{\mathrm{G}}$-nitro-L-arginine methyl ester (L-NAME). In cardiac tissue thiobarbituric acid reactive substances (TBARS) concentration, reduced glutathione (GSH) content, endothelial NOS (eNOS), and Akt expression were also measured. The blueberry extract showed higher total phenols and anthocyanins contents, scavenging activity of superoxide radical and systolic and diastolic function was improved, TBARS diminished, GSH was partially preserved, and both NOS and Akt expression increased in hearts treated with the extract. These beneficial effects were lost when eNOS was inhibited. In resume, these data show that the increase of eNOS expression via Akt and the scavenging activity contribute to the cardioprotection afforded by acute treatment with Colombian blueberry extract against ischemia and reperfusion injury.
\end{abstract}

\section{Introduction}

Oxidative stress is defined as the imbalance between the generation of reactive oxygen species (ROS) and antioxidant defense mechanisms. Myocardial damage induced by ischemia-reperfusion of the heart has been proposed to be caused, at least in part, by ROS production [1-3]. Indirect evidence supporting this hypothesis has been the cardioprotective effects of ROS scavengers and agents capable of inducing antioxidants such as glutathione peroxidase (GSHpx) and superoxide dismutase (SOD) and of supplementing antioxidants [4]. The detrimental action of ROS has naturally led to increased interest in antioxidants, in particular, plant diet-derived antioxidants, as possible therapeutic agents [5, 6]. Unfortunately, the attempts to use synthetic antioxidants to block or attenuate the harmful effects of ROS have produced mixed and mostly negative results $[7,8]$. There are various elements involved in this "antioxidant paradox," such as the selection of dose, degree of collateral blood flow, duration of ischemia, timing of drug administration, and drug delivery method [9]. Reduced nitric oxide (NO) bioavailability is other important factor mediating ischemia and reperfusion injury [10]. Thus, the treatment with NO donors is able to prevent reperfusion-induced cell death 
[11]. Data from our group [12] and other laboratories [13] show that NO mediates the beneficial effects of natural antioxidants.

The polyphenols present in fruits, green tea, "yerba mate," black chocolate, or red wine exert antioxidant properties through various mechanisms of action including the ROS scavenging and ROS generation inhibition, thereby preventing damage to lipids, proteins, nucleic acids, and eventually cell damage and death [14]. These properties appear involved in the beneficial effects of those natural products on health previously reported by us [12, 15-17] and other researchers $[18,19]$. The genus Vaccinium provides a group of plants with promising antioxidant properties. It includes about 400 species, which grow principally in the hillside of tropical mountains. One of them, agraz or Colombian blueberry, is the species Vaccinium meridionale Swartz (Ericaceae) which grows in the Andean region of South America at 2300$3300 \mathrm{~m}$ and it is a wild bush of 1-4 m high, sometimes until $8 \mathrm{~m}$. The fruits are edible dark purple berries [20]. It is currently available only in the wild and their spontaneous populations are found across the Colombian mountains [21]. Blueberries contain phenolics such as anthocyanins and flavonoids and appear to have the highest antioxidant capacity among fruits and vegetables [22, 23], and the fermented juice, such as in the case of wine, improves its processing and storage effects on anthocyanin composition, color, and appearance [24]. Thus, consumption of berries or their related products could be of importance not only in the maintenance of health $[25,26]$ but also in the preventing of cardiovascular disease. Thus, Ahmet et al. [27] showed that a blueberryenriched diet increased myocardial tolerance to ischemic damage in a rat model of postmyocardial infarction dilated cardiomyopathy.

Therefore, the objective of the current study was to assess the antioxidant properties and the effects against reperfusion injury in the isolated rat heart of a fermented nonalcoholic extract of Colombian blueberry, examining the role played by NO.

\section{Materials and Methods}

2.1. Plant Material. Vaccinium meridionale constitutes the raw material used for the fermentation in this research; berries fruits were harvested at the beginning of December 2009, in Colombia, zone "El Retiro" (2175 m.a.s.l) into Antioquia region. The plant and berries were identified by the Corporación Colombiana de Investigación Agropecuaria (Corpoica), La Selva, Colombia (voucher specimen number ILS 14050070). The berries were manually harvested at the commercially appropriate ripeness stage (fruit maturity was based on fruit surface color) and transported to the Laboratory of Food Science, Faculty of Sciences, National University of Colombia in hermetic plastic canisters. In this place, the berries were cleaned by removing leaves, stems, and unripe and damaged berries.

2.2. Blueberries Processing. The maceration was performed in the traditional fashion, punching the berries fruits $(2 \mathrm{~kg})$ several times at $-18^{\circ} \mathrm{C}$ during 2 days. After prefermentative cold maceration, $\mathrm{H}_{2} \mathrm{O}$ purified $(2 \mathrm{~L})$ was added.

2.3. Fermentation Experiment. The berry juice was transferred into stainless steel tank $(5 \mathrm{~L}, 320 \times 144 \times 2 \mathrm{~mm})$, and the fermentation was carried out by the inoculation of $0.165 \mathrm{~g} / \mathrm{L}$ of selected cultures of yeast Saccharomyces cerevisiae Meyen ex E.C. Hansen 1883 (ARS Culture Collection, USDA). The fermentation was performed at $25 \pm 2^{\circ} \mathrm{C}$ for 10 days. The soluble solids were adjusted to $30^{\circ} \mathrm{Brix}$ with sugar. The fermentation was stopped by the addition of $40 \mathrm{mg} \mathrm{SO} / 2$. Then, the fermented berry product was decanted, treated with albumin $(0.4 \mathrm{~g} / \mathrm{L})$, and filtered through filter plates $(45 \mu \mathrm{m})$.

2.4. Standard Chemical Analysis. The total titratable acidity was assessed by titration with sodium hydroxide $(0.1 \mathrm{~N})$ and expressed as tartaric acid. The $\mathrm{pH}$ value was measured using a digital $\mathrm{pH}$ meter (Hanna Instruments, Buenos Aires, Argentina). Total soluble solids ( ${ }^{\circ}$ Brix) were measured as Brix using a refractometer RX-5000 (Atago, Tokyo, Japan). The alcoholic title was quantified as ethanol by GC-FID, using an Agilent 6890N GC system (Ramsey, MN, USA). The values of these parameters at the beginning and at the end of fermentation process are showed in Table 1. HPLC analysis was carried out using a column Zorbax C18 $(250 \times 4.6 \mathrm{~mm}$, $5 \mu \mathrm{m}$ particle size), oven temperature $38^{\circ} \mathrm{C}$. The solvents used were acetonitrile/formic acid/water $(3: 10: 87)$ as solvent $\mathrm{A}$ and acetonitrile/formic acid/water $(50: 10: 40)$ as solvent $\mathrm{B}$. The elution gradient was as follows: 0 min $94 \% \mathrm{~A}$ and $6 \% \mathrm{~B}$; $10 \mathrm{~min} 80 \% \mathrm{~A}$ and $20 \% \mathrm{~B} ; 20 \mathrm{~min} 60 \% \mathrm{~A}$ and $40 \% \mathrm{~B} ; 30 \mathrm{~min}$ $50 \% \mathrm{~A}$ and $50 \% \mathrm{~B} ; 35 \mathrm{~min} 94 \% \mathrm{~A}$ and $6 \% \mathrm{~B}$. The flow rate was $0.8 \mathrm{~mL} / \mathrm{min}$. Detection was measured at $525 \mathrm{~nm}$. All analyses were made in triplicate.

2.5. Preparation of the Nonalcoholic Extract (BE). The extract of berries fermented $(200 \mathrm{~mL})$ was vacuum-evaporated $\left(<30^{\circ} \mathrm{C}\right)$. The berry juice $(\mathrm{BJ})$ was used as control. After being filtered, the aqueous extract was lyophilized and dry matter was maintained at $-20^{\circ} \mathrm{C}$ until used. The nonalcoholic extract was chromatographed by HPLC and a representative chromatogram is included in Figure 1. The solid residue was 0.04 and $0.18 \mathrm{~g} / \mathrm{mL}$ for $\mathrm{BE}$ and $\mathrm{BJ}$, respectively. The residues were solubilized in deionized or Ringer's solution immediately before the assays.

2.6. Determination of Total Phenols and Total Anthocyanins Contents. Total phenolic content was measured by using the Folin-Ciocalteu. Results were expressed as caffeic acid equivalents/mg of dry weight of the extract [28]. Total anthocyanins were determined by using a $\mathrm{pH}$ differential method [29]. Results were expressed as mg of cyanidin-3glucoside equivalents/mg of dry weight of the extract.

\subsection{Total Antioxidant Activity}

2.7.1. 1,1-Diphenyl-2-picryl-hydrazyl (DPPH) Scavenging Activity. Reduction of the stable free radical DPPH was determined with the aid of a modified version of the method 


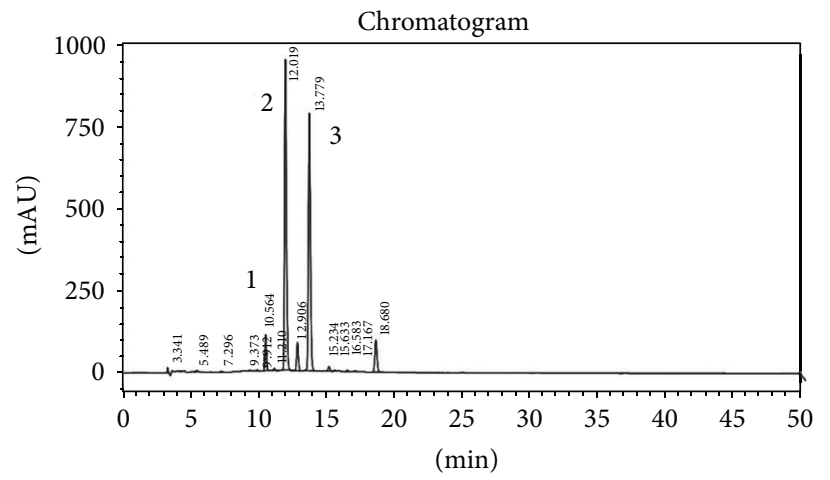

(a)

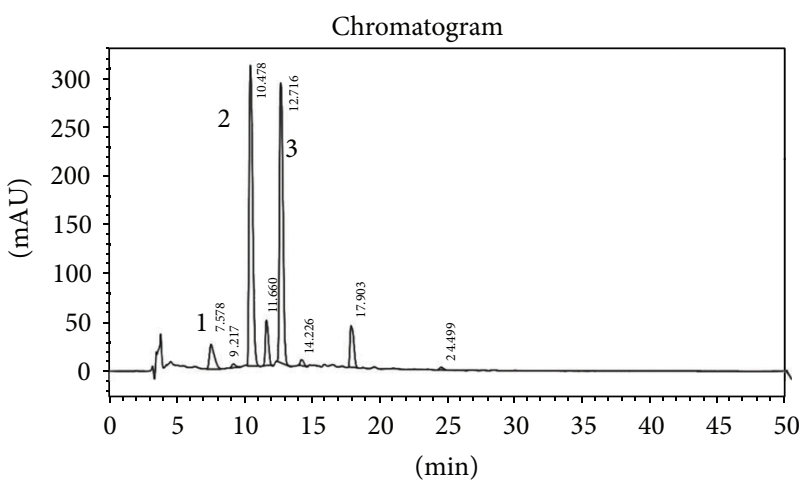

(b)

FIGURE 1: Anthocyanins profile. HPLC-UV chromatograms of fermented blueberry, (a) corresponds to the anthocyanins profile at the beginning of the fermentation process and (b) corresponds to the anthocyanins profile at the end of the fermentation process. Peak identification: 1, Delphinidin 3-glucoside; 2, cyanidin-3-glucoside; 3, petunidin-3-glucoside. Chromatographic analysis was carried out using a column Zorbax C18 and mixtures of acetonitrile/formic acid/water as mobile phase. The flow rate was $0.8 \mathrm{~mL} / \mathrm{min}$ and detection was measured at $525 \mathrm{~nm}$.

TABLE 1: Physicochemical characteristics of berry process fermentation.

\begin{tabular}{lcc}
\hline Days & 1 & 10 \\
Treatment & $\mathrm{BE}$ & $\mathrm{BE}$ \\
Ethanol \% $^{(\mathrm{a})}$ & 0.20 & 11.05 \\
$\mathrm{pH}$ & 2.58 & 2.82 \\
${ }^{\circ} \mathrm{Brix}$ & 30 & 6.2 \\
Total titratable acidity $^{(\mathrm{b})}$ & 7.01 & 8.89 \\
\hline
\end{tabular}

${ }^{(a)}$ Expressed as alcoholic title \% w/v; ${ }^{\left({ }^{b}\right)}$ Expressed as g/L of tartaric acid. BE: berry extract.

described by Cavin et al. [30]. The results are expressed in $\mu \mathrm{g}$ caffeic acid equivalents/mg of dry weight of the extract.

\subsubsection{2,2'-Azino-bis(3-ethylbenzothiazoline-6-sulphonic Acid} $(A B T S)^{\circ+}$ Scavenging Activity. Reduction of the stable ABTS radical cation was determined with the aid of a modified version of the method described by Re et al. [31]. Results are expressed as caffeic acid equivalents/mg of dry weight of the extract.

2.7.3. Ferric Reducing Antioxidant Power (FRAP). The FRAP assay was carried out to determine the reducing ability of the extracts with a method adapted from Benzie and Strain [32]. Results were expressed as $\mu \mathrm{mol}$ of ascorbic acid equivalents/mg of dry weight of the extract.

\subsection{Scavenging Activities}

2.8.1. Scavenging of Superoxide Anion $\left(\mathrm{O}_{2}{ }^{--}\right) \cdot \mathrm{O}_{2}{ }^{--}$was generated through enzymatic oxidation of hypoxanthine with xanthine oxidase grade I $(0.06 \mathrm{U})$ and was detected by the reduction of nitroblue-tetrazolium (NBT), monitored spectrophotometrically at $560 \mathrm{~nm}$. Details of this assay have been described elsewhere [17]. Allopurinol was used as a reference.
2.8.2. Scavenging of Peroxynitrite Anion $\left(\mathrm{ONOO}^{-}\right) . \mathrm{ONOO}^{-}$ was synthesized in a quenched flow reactor in accordance with the method described by Koppenol et al. [33]. The pyrogallol red bleaching assay was carried out as reported by Balavoine and Geletii [34].

2.8.3. Nitration of Blood Serum Albumin (BSA) Induced by $\mathrm{ONOO}^{-}$. Reaction between $\mathrm{ONOO}^{-}$and BSA was carried out as described by Jiao et al. [35]. Samples of control, BE, and BJ were analyzed by SDS-polyacrylamide gel electrophoresis (SDS-PAGE, 10\%) following the Laemmli method by staining with Coomasie blue. The nitration of BSA was examined by the Western blot using an antinitrotyrosine polyclonal antibody (Cayman Chemicals, Ann Arbor, MI, USA). The bands were visualized with an ECL-Plus chemiluminescence detection system and analyzed by densitometric analysis using Scion Image software (Scion Co., Frederick, MD, USA).

2.8.4. Hydroxyl Radical $\left(\mathrm{OH}^{\circ}\right)$ Scavenging Assay. The $\mathrm{OH}^{*}$ scavenging assay was carried out according to the method described by Schinella et al. [17]. This assay was performed in triplicate with DMSO $(20 \mathrm{mM})$ as a reference.

2.9. Isolated Heart Preparation. All procedures followed during this investigation conform to the Guide for the Care and Use of Laboratory Animals published by the US National Institutes of Health (NIH Publication No. 85-23, revised 1996) and to the guidelines laid down by the Animal Welfare Committee of La Plata School of Medicine. Male Wistar rats of 5-6 months of age were anesthetized with an intraperitoneal injection of sodium pentobarbital $(60 \mathrm{mg} / \mathrm{kg}$ body $\mathrm{wt})$. The heart was excised and perfused through the aorta by the nonrecirculating Langendorff technique with Ringer's solution $(\mathrm{pH} 7.4)$ and at $37^{\circ} \mathrm{C}$. Heart rate was maintained at $280 \pm 10$ beats $/ \mathrm{min}$. A latex balloon was placed inside the left ventricle (LV) and connected to a Statham P23XL pressure transducer. The balloon was filled with water to provide an end-diastolic pressure (LVEDP) of $8-12 \mathrm{mmHg}$, 
and this volume was unchanged for the rest of the experiment. Coronary perfusion pressure was adjusted to approximately $60-70 \mathrm{mmHg}$ and coronary flow was $11 \pm 2 \mathrm{~mL} / \mathrm{min}$. Left ventricular pressure (LVP) was acquired by using an analogto-digital converter and acquisition software (Chart V4.2.3 AD Instruments Sudamérica, Santiago de Chile, Chile).

2.9.1. Experimental Protocols. After $10 \mathrm{~min}$ of stabilization, the following experimental protocols were performed. Ischemic control (IC) hearts $(n=6)$ : hearts were subjected to $20 \mathrm{~min}$ of normothermic global ischemia followed by $30 \mathrm{~min}$ of reperfusion. Global ischemia was induced by stopping the perfusate inflow line and the heart was placed in a saline bath held at $37^{\circ} \mathrm{C}$. Hearts treated with BE and BJ ( $n=6$ for each treatment): hearts were treated $10 \mathrm{~min}$ before ischemia and the initial $10 \mathrm{~min}$ of reperfusion with the blueberry extract $(50 \mu \mathrm{g} / \mathrm{mL})$. The administration time was selected taking into account that reperfusion injury is the result of alterations that occur during ischemia and the first minutes of reperfusion [16]. Other hearts $(n=4)$ received $1 \mathrm{mM}$ of $\mathrm{N}^{\mathrm{G}}$-nitroL-arginine methyl ester (L-NAME), a nonselective NOS inhibitor, $20 \mathrm{~min}$ before ischemia and during the reperfusion period. The L-NAME administration was extended through the entire reperfusion time to prevent the activation of the different NOS isoforms.

2.9.2. Systolic and Diastolic Function. Myocardial contractility was assessed through the left ventricular developed pressure (LVDP), obtained by subtracting LVEDP to the LVP peak values and the maximal rise velocity of the left ventricular pressure $\left(+d P / d t_{\max }\right)$. Data were expressed as percentages of their respective preischemic values. The diastolic function was evaluated through the isovolumic LVEDP.

2.10. Biochemical Determinations from Isolated Heart Preparation. At the end of reperfusion, hearts were frozen in liquid $\mathrm{N}_{2}$ and kept at $-70^{\circ} \mathrm{C}$ until the moment of assays.

2.10.1. Assessment of Lipid Peroxidation. A portion of LV was homogenized in a solution composed by $25 \mathrm{mM} \mathrm{K \textrm {K } _ { 2 }} \mathrm{PO}_{4}$ $140 \mathrm{mM} \mathrm{KCl}$ at $\mathrm{pH}=7.4$ with a Polytron homogenizer. The samples were centrifuged and in the supernatant, an index of lipid peroxidation TBARS was determined by a spectroscopic technique and expressed as nmol MDA/g tissue weight [36].

2.10.2. Reduced Glutathione (GSH). Aliquots of homogenate were used to assess reduced glutathione content (GSH) according to Ellman's method [37] and expressed as nmol $\mathrm{GSH} / \mathrm{g}$ tissue weight.

2.10.3. Western Blot Analysis. Other portion of LV was homogenized in ice-cold RIPA buffer $(300 \mathrm{mM}$ sucrose, $1 \mathrm{mM}$ DTT, $4 \mathrm{mM}$ EGTA, $20 \mathrm{mM}$ Tris $\mathrm{pH} 7.4,1 \%$ Triton $\mathrm{X}$, $10 \%$ protease cocktail, $25 \mu \mathrm{M} \mathrm{NaF}$, and $1 \mu \mathrm{M}$ orthovanadate), centrifuged at $10000 \times \mathrm{g}$ for $15 \mathrm{~min}$ at $4^{\circ} \mathrm{C}$. The supernatant was collected and subjected to SDS-PAGE. The samples were transferred to a PVDF membrane (2 h). Equal loading of samples was confirmed by Ponceau S staining. Membranes were blocked with $5 \%$ nonfat milk in Tris-buffered saline ( $\mathrm{pH} 7.5$ ) containing $0.1 \%$ Tween (TBS-T) and probed overnight at $4^{\circ} \mathrm{C}$ with antibodies anti-eNOS (Sigma-Aldrich, St. Louis, MO, USA), anti-Akt (Calbiochem, Merck Millipore Darmstadt, Germany). Membranes were washed four times for $10 \mathrm{~min}$ in TBS-T prior to the addition of anti-rabbit secondary antibody (1:1000 dilution) and the antibody-antigen complexes were developed using a chemiluminescent system (ECL Plus; GE Healthcare, Buckinghamshire, UK).

2.11. Statistical Analysis. Data were expressed as means $\pm \mathrm{SE}$. Statistical analysis was performed by one-way analysis of variance (ANOVA) followed by Student-Newman-Keuls multiple comparisons test. Differences were considered significant at $P<0.05$.

\section{Results}

3.1. Antioxidant Activity. The total antioxidant activity was measured through the ability of the extracts to reduce $\mathrm{DPPH}^{\bullet} \mathrm{ABTS}^{*}$ and $\mathrm{Fe}^{3+}$ - and the content of total phenols and anthocyanins of $\mathrm{BE}$ was higher than $\mathrm{BJ}$. BE also showed a higher $\mathrm{O}_{2}^{--}$and $\mathrm{ONOO}^{-}$scavenging activity in comparison to BJ (Table 2). Moreover, it was detected as an attenuation of BSA degradation (Figure 2(a)) and nitration (Figure 2(b)) in the presence of BE. As indicated by graphic bar BE and BJ significantly decreased the percentage of nitrotyrosine compared to control (Figure 2(b)). However, the change produced by BJ was lesser to that obtained by BE. At a final concentration of $100 \mu \mathrm{g} / \mathrm{mL}$, none of the extracts were able to scavenge the $\mathrm{OH}^{\circ}$ produced in the $\mathrm{Fe}^{3+}$-EDTA $+\mathrm{H}_{2} \mathrm{O}_{2}$ system in the presence or the absence of ascorbate. Moreover, the results showed a significant increase of deoxyribose degradation after the treatment with the two samples in the absence of ascorbate (data not shown).

3.2. Myocardial Systolic and Diastolic Function and Oxidative Damage of Cardiac Tissue. After BE treatment the postischemic recovery improved, reaching LVDP values significantly higher than that obtained in IC hearts $(87 \pm 8 \%$ versus $42 \pm 3 \%$ at the end of reperfusion period). The treatment with $\mathrm{BJ}$ did not modify the postischemic recovery detected in IC hearts and at the end of reperfusion LVDP reached a value of $39 \pm 9 \%$ (Figure 3(a)). A similar pattern was obtained when $+d P / d t_{\max }$ was analyzed (Figure $3(\mathrm{~b})$ ). The ischemic contracture observed in IC hearts was not modified after all the treatments applied. The treatment with BE but not with BJ was able to attenuate the increase of LVEDP detected during reperfusion in IC hearts $(18 \pm 6 \mathrm{mmHg}$ versus $49 \pm 6 \mathrm{mmHg}$ at the end of reperfusion period) (Figure 3(c)). The NOS inhibition with L-NAME abolished the protective effect of BE on postischemic recovery of systolic myocardial function (Figure 4). In addition, LVEDP was impaired after the combination of $\mathrm{BE}+\mathrm{L}-\mathrm{NAME}$ reaching a value of $59 \pm$ $14 \mathrm{mmHg}$ at the end of the reperfusion period.

TBARS content (Figure 5(a)) diminished and GSH level (Figure 5(b)) was partially preserved when hearts were treated with BE but not after BJ compared with untreated hearts. These beneficial effects were annulled in presence of NOS inhibition detecting an increase of lipid peroxidation 
TABLE 2: Phenolics compounds, total antioxidant activity, and reactive oxygen species (ROS) scavenging properties of fermented berry extract (BE) and berry juice (BJ).

\begin{tabular}{|c|c|c|c|c|c|c|c|}
\hline \multirow{2}{*}{ Sample } & \multicolumn{2}{|c|}{ Phenolic compounds } & \multicolumn{3}{|c|}{ Antioxidant activity } & \multicolumn{2}{|c|}{ ROS scavenging } \\
\hline & Total phenolics ${ }^{(\mathrm{a})}$ & Total anthocyanins $s^{(\mathrm{c})}$ & $\mathrm{DPPH}^{(\mathrm{a})}$ & $\mathrm{ABTS}^{\bullet+(\mathrm{a})}$ & FRAP $^{(b)}$ & $\mathrm{O}_{2}^{\cdot-(\mathrm{a})}$ & $\mathrm{ONOO}^{-(\mathrm{b})}$ \\
\hline $\mathrm{BE}$ & 49 & 34 & 23 & 73 & 220 & 5.6 & 5.0 \\
\hline BJ & 18 & 12 & 5 & 24 & 72 & 2.3 & 1.3 \\
\hline
\end{tabular}

${ }^{(a)}$ Results are expressed as caffeic acid equivalent ( $\mu \mathrm{g} / \mathrm{mg}$ dry weight of extract); ${ }^{(b)}$ results are expressed as ascorbic acid equivalent $(\mu \mathrm{mol} / \mathrm{mg}$ dry weight of extract); ${ }^{(c)}$ results are expressed as cyanidin-3-glucoside equivalents $(\mu \mathrm{mol} / \mathrm{mg}$ dry weight of extract).

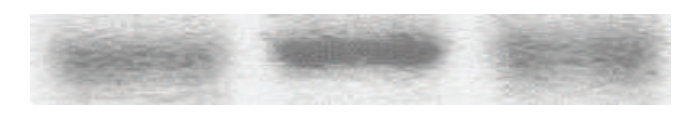

(a)

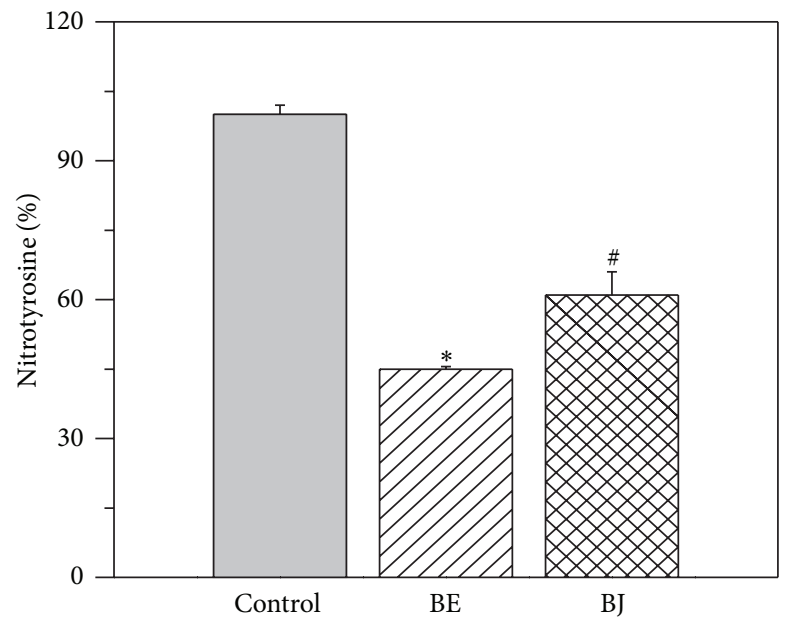

(b)

FIgURE 2: (a) Degradation; (b) nitration of BSA induced by $\mathrm{ONOO}^{-}$. Both extracts attenuated the degradation and nitration of BSA compared to control. However, the effects of fermented berry extract (BE) were significantly higher than that of berry juice (BJ). ${ }^{*} P<0.05$ with respect to control; ${ }^{*} P<0.05$ with respect to BE.

and less GSH level (Figures 5(a) and 5(b): BE + L-NAME column).

The treatment with BE increased the expression of eNOS compared to that detected in IC hearts while BJ treatment decreased it (Figure 6(a)). When BE was administered in the presence of NOS inhibitor L-NAME, eNOS expression decreased to levels significantly lesser than those of IC. The expression of p-Akt (Figure 6(b)) showed the same changes except in the BJ group in which the p-Akt expression was not different to IC hearts.

\section{Discussion}

The present study provides evidence about the antioxidant properties and cardioprotective effects of a nonalcoholic extract of fermented Colombian blueberry in isolated rat heart. This fermented extract showed higher antioxidant capacity (Figure 2 and Table 2) in comparison to BJ attributed to their higher content of total phenols and anthocyanins. It also detected a different composition of polyphenols (Figure 1) in BE in comparison to BJ.

In isolated rat hearts, the treatment with $\mathrm{BE}$ improved the postischemic recovery of systolic and diastolic myocardial function. Therefore, an increase of contractility parameters as left ventricular developed pressure (Figure 3(a)) and the maximal rise velocity of the left ventricular pressure $\left(+d P / d t_{\max }\right.$, Figure 3(b)) were detected in treated hearts when the coronary flow was restored. Simultaneously, a decrease of left ventricular end-diastolic pressure was observed during reperfusion in hearts treated with BE indicating that myocardial diastolic stiffness was minor in the presence of the extract (Figure 3(c)). The figure also shows that the treatment with BJ did not improve the postischemic recovery of myocardial function displaying the absence of beneficial effects in our experimental conditions.

Which are the mechanisms involved in the cardioprotective action of BE? The NO derived principally of the constitutive endothelial nitric oxidase synthase (eNOS) is able to exert beneficial or deleterious effects. Thus, NO interacts with proteins via S-nitrosylation at specific cysteine residues to alter their function $[38,39]$ which has an important meaning regarding the excitation-contraction (EC)-coupling-related process [38]. In the normal range of $\mathrm{O}_{2}{ }^{--}$, S-nitrosylation is accommodated; however, it is inhibited when $\mathrm{O}_{2}{ }^{--}$is increased [40]. As noted, increased $\mathrm{O}_{2}{ }^{--}$interacts with $\mathrm{NO}$ to form $\mathrm{ONOO}^{-}$, a reactive species that is capable of triggering 


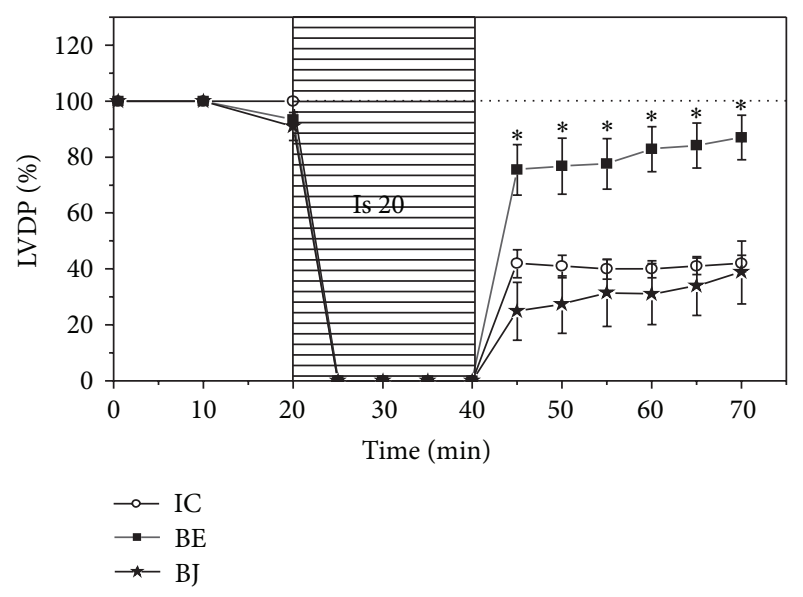

(a)

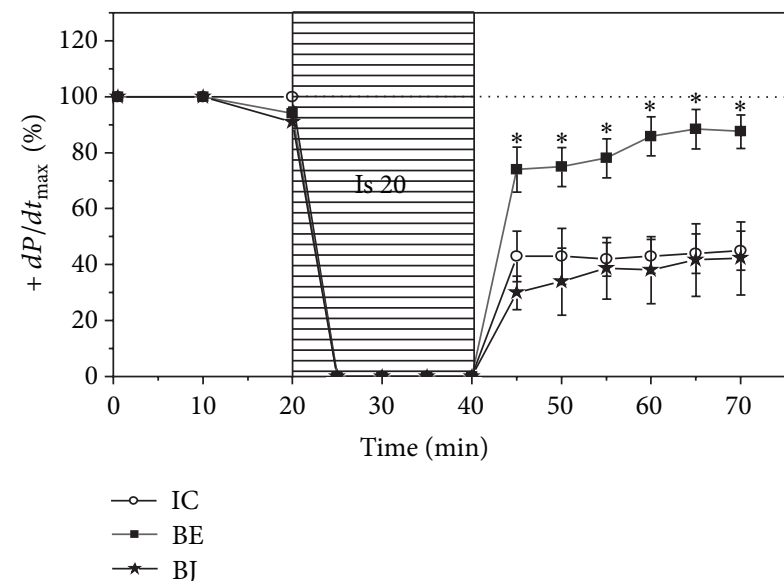

(b)

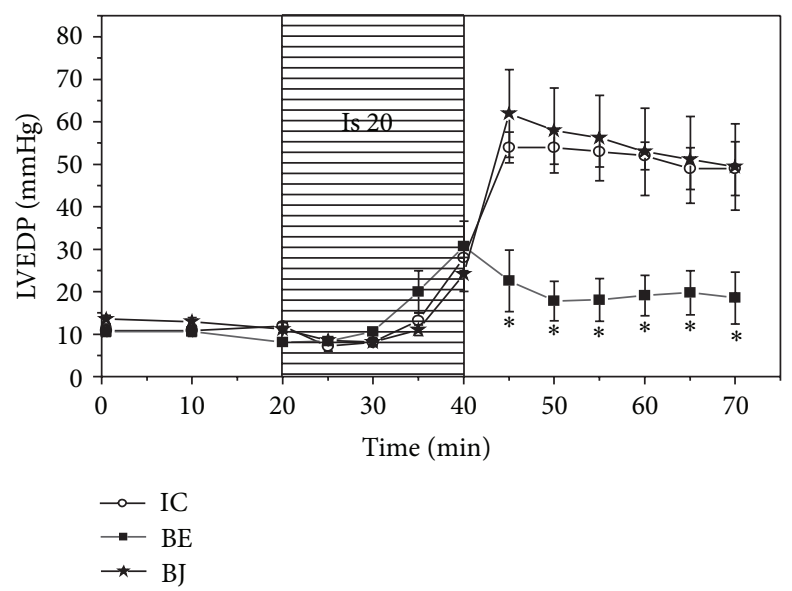

(c)

FIGURE 3: (a) Time course of left ventricular developed pressure (LVDP); (b) maximal rise velocity of left ventricular pressure (+dP/dt $t_{\text {max }}$ ); left ventricular end-diastolic pressure (LVEDP) during ischemia and reperfusion in ischemic control hearts (IC, $n=6)$ and in hearts treated with the fermented berry extract $(\mathrm{BE}, n=6)$ and berry juice $(\mathrm{BJ}, n=6)$. Note that LVDP and $+d P / d t_{\max }$ increased and LVEDP decreased during reperfusion only after treatment with $\mathrm{BE} .{ }^{*} P<0.05$ with respect to IC.

an array of cytotoxic processes, including lipid peroxidation, protein oxidation, and nitration (altering EC coupling) [41, 42]. In our experiments, the eNOS inhibition abolished the cardioprotective effects afforded by BE. Thus, an impairment of systolic and diastolic myocardial function was evident in the presence of L-NAME (Figure 4) indicating that NO plays a crucial role in the attenuation of contractile dysfunction that follows the ischemia and the reperfusion.

On the other hand, it is well known that brief episodes of myocardial ischemia and reperfusion are associated with the generation of ROS [1-3,43]. ROS cause lipid peroxidation first leading to reversible damage and eventually to necrosis and/or apoptosis. The administration of antioxidants or free radicals scavengers is able to limit the evolution of myocardial damage reducing ROS-induced lipid peroxidation [44]. In this study, we found that TBARS decreased and the level of GSH was higher in hearts treated with BE in comparison to ischemic control hearts displaying an attenuation of oxidative stress by the extract. However, lipid peroxidation increased and GSH levels decreased in hearts treated with L-NAME, showing the predominance of ROS and the consequent oxidative damage when eNOS was inhibited (Figures 5(a) and 5(b)). Western blot analysis revealed that eNOS expression of hearts treated with $\mathrm{BE}$ increased compared to untreated hearts and this effect was annulled by eNOS inhibition (Figure 6(a)). Which can be the pathway of eNOS activation by BE? As shown in Figure 6(b), the level of Akt phosphorylation also increased in hearts treated with $\mathrm{BE}$, effect that was abolished by eNOS inhibition. Taking into account that phosphatidylinositol-3-kinase- (PI3K-) Akt can activate eNOS [45], our data suggest that Akt is involved in the activation of eNOS mediated by BE.

Because TBARS decrease coincides with higher eNOS expression level and that L-NAME treatment prevented these changes, it might at first be assumed that eNOS-derived NO could be involved in the attenuation of oxidative stress that occurs during ischemia and reperfusion. In fact, a close relationship between $\mathrm{NO}$ and oxidative stress has long been proposed [46]. Moreover, data from Clancy et al. [47] indicate that $\mathrm{NO}$ reacts with intracellular GSH forming 


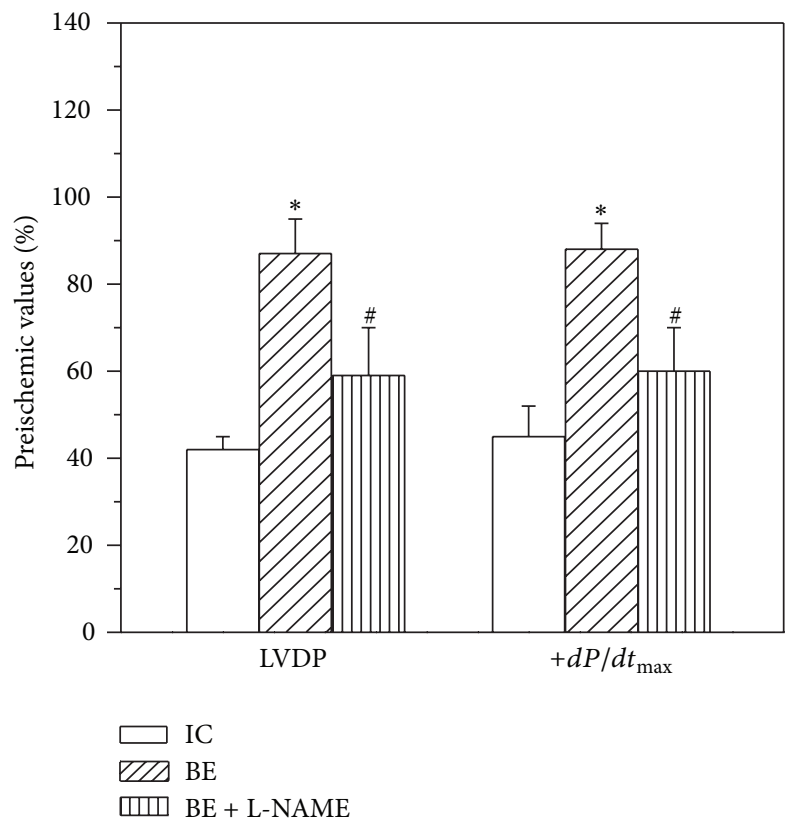

FIGURE 4: Values of left ventricular developed pressure (LVDP) and maximal rise velocity of left ventricular pressure $\left(+d P / d t_{\max }\right)$ at the end of reperfusion period in ischemic control hearts (IC, $n=6)$ and in hearts treated with fermented berry extract $(\mathrm{BE}, n=6)$ and BE $+\mathrm{L}-\mathrm{NAME}$ (NOS inhibitor) $(n=4)$. Note that L-NAME (NOS inhibitor) significantly attenuated the improvement of LVDP and $+d P / d t_{\max }$ observed after BE treatment. ${ }^{*} P<0.05$ with respect to IC; ${ }^{\#} P<0.05$ with respect to $\mathrm{BE}$.

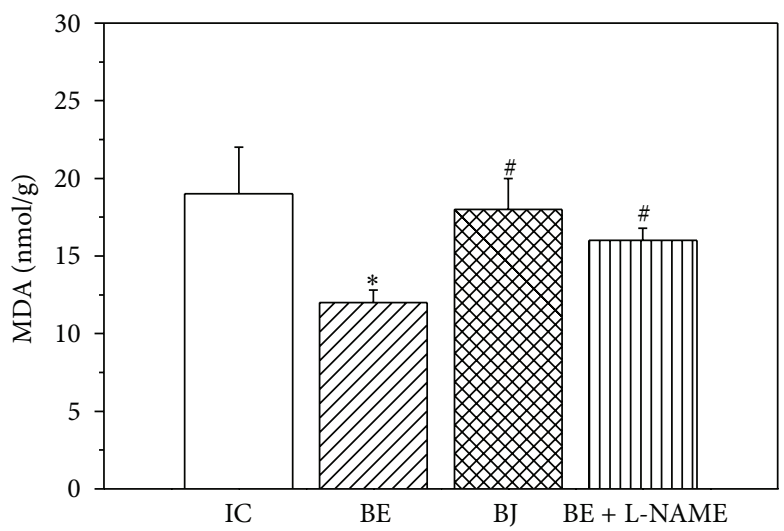

(a)

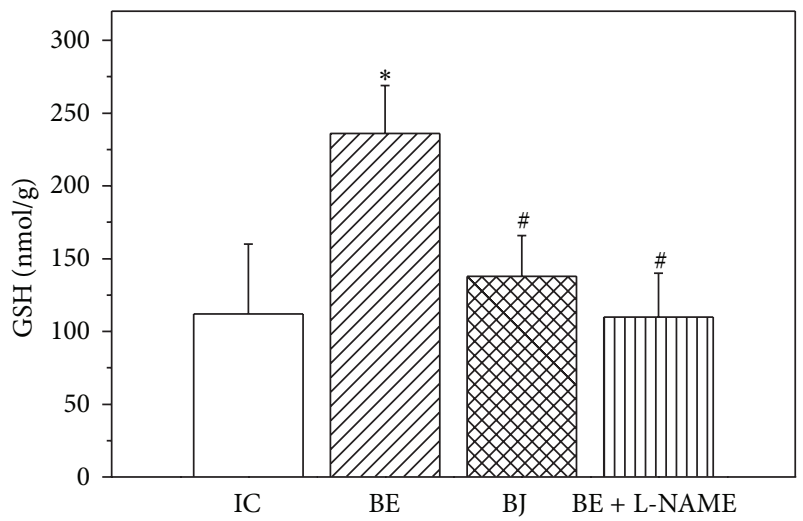

(b)

FIGURE 5: (a) Effects of fermented berry extract (BE, $n=6)$ and berry juice (BJ, $n=6)$ and BE + L-NAME $(n=4)$ on lipid peroxidation (MDA); (b) reduced glutathione (GSH). The treatment with BE attenuated lipid peroxidation and preserved GSH levels in comparison to IC hearts. These favorable changes were annulled when BE was administered in the presence of NOS inhibitor, L-NAME. ${ }^{*} P<0.05$ with respect to $\mathrm{IC} ;{ }^{\#} P<0.05$ with respect to $\mathrm{BE}$.

S-nitrosoglutathione, compound which may function as stable intermediate of NO activity. Therefore, the improvement of GSH level added to the in vitro demonstration of the capacity of the extract to remove $\mathrm{O}_{2}{ }^{--}$and $\mathrm{ONOO}^{-}$could be indicating the presence of a higher NO bioavailability in hearts treated with $\mathrm{BE}$ compared to untreated hearts. Therefore, these data are strong arguments to justify the involvement of $\mathrm{NO}$ in the attenuation of postischemic myocardial dysfunction afforded by the extract.
This study clearly demonstrates for the first time the protective effect of a nonalcoholic extract of berry fruit from Vaccinium meridionale against reperfusion injury in isolated rat heart. These cardioprotective properties may be linked to the ability of the extract to induce eNOS expression via Akt and to scavenge ROS. From these results, we propose that the "mortiño" extract could be a promising therapeutic approach against myocardial dysfunction. Moreover, being the fermented extract of berry fruit a source of polyphenols, 


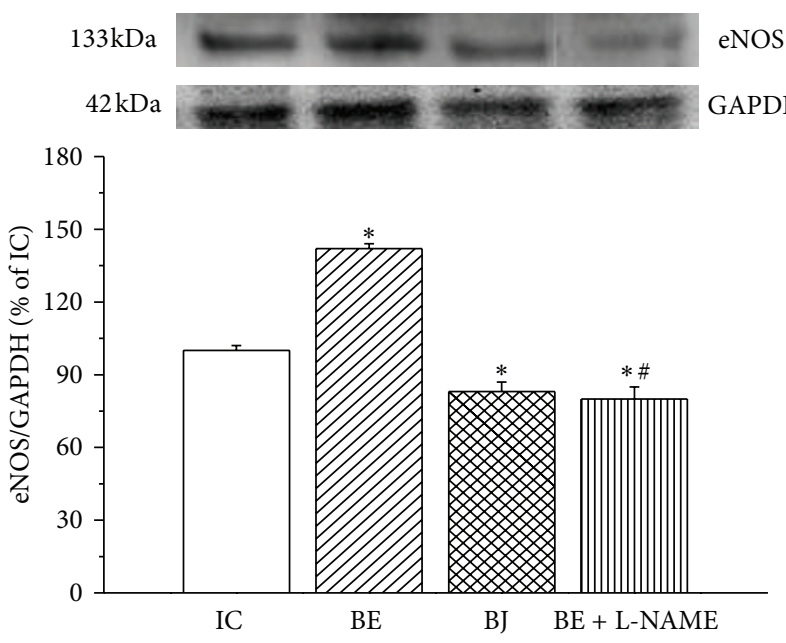

(a)

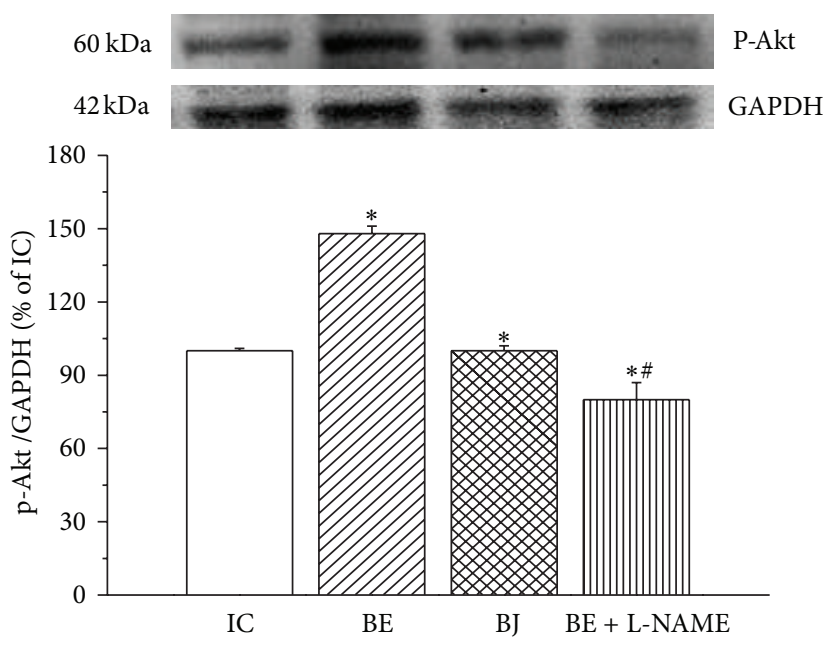

(b)

Figure 6: (a) Representative Western blots and mean data of eNOS; (b) phospho-Akt (p-Akt) protein expression normalized to GAPDH in ischemic control hearts (IC, $n=6)$ and in hearts treated with both fermented berry extract $(\mathrm{BE}, n=6)$ and berry juice $(\mathrm{BJ}, n=6)$ and $\mathrm{BE}$ $+\mathrm{L}-\mathrm{NAME}(n=4)$. It is observed that only BE increased the expression of eNOS and p-Akt and this increase was abolished when NOS was inhibited with L-NAME. ${ }^{*} P<0.05$ with respect to IC; ${ }^{\#} P<0.05$ with respect to $\mathrm{BE}$.

it could be used as a functional food to prevent or mitigate pathologies associated to oxidative stress [48].

\section{Authors' Contribution}

G. Schinella and S. Mosca collaborated equally to the present work.

\section{Acknowledgments}

The authors thank Daniel Fioravanti for excellent technical assistance. This study was supported by the Grant M-139 from the National University of La Plata of Argentina to Dr. S. Mosca and the Spanish government (MICIIN and FEDER, Grant SAF2009-10059-C03-01). Y. E. Lopera thanks the grant received from COLCIENCIAS.

\section{References}

[1] M. L. Weisfeldt, J. Zweier, G. Ambrosio, L. C. Becker, and J. T. Flaherty, "Evidence that free radicals result in reperfusion injury in heart muscle," Basic Life Sciences, vol. 49, pp. 911-919, 1988.

[2] G. Ambrosio, J. L. Zweier, C. Duilio et al., "Evidence that mitochondrial respiration is a source of potentially toxic oxygen free radicals in intact rabbit hearts subjected to ischemia and reflow," The Journal of Biological Chemistry, vol. 268, no. 25, pp. 18532$18541,1993$.

[3] K. K. Griendling and G. A. FitzGerald, "Oxidative Stress and cardiovascular injury part I: Basic mechanisms and in vivo monitoring of ROS," Circulation, vol. 108, no. 16, pp. 1912-1916, 2003.

[4] N. Marczin, N. El-Habashi, G. S. Hoare, R. E. Bundy, and M. Yacoub, "Antioxidants in myocardial ischemia-reperfusion injury: therapeutic potential and basic mechanisms," Archives of Biochemistry and Biophysics, vol. 420, no. 2, pp. 222-236, 2003.
[5] O. I. Aruoma, T. Bahorun, and L. S. Jen, "Neuroprotection by bioactive components in medicinal and food plant extracts," Mutation Research, vol. 544, no. 2-3, pp. 203-215, 2003.

[6] D. Bandyopadhyay, A. Chattopadhyay, G. Ghosh, and A. G. Datta, "Oxidative stress-induced ischemic heart disease: protection by antioxidants," Current Medicinal Chemistry, vol. 11, no. 3, pp. 369-387, 2004.

[7] L. S. Cozma, "The role of anti-oxidant therapy in cardiovascular disease," Current Opinion in Lipidology, vol. 15, no. 3, pp. 369371, 2004.

[8] C. A. Papaharalambus and K. K. Griendling, "Basic mechanisms of oxidative stress and reactive oxygen species in cardiovascular injury," Trends in Cardiovascular Medicine, vol. 17, no. 2, pp. 48-54, 2007.

[9] B. Halliwell, "The wanderings of a free radical," Free Radical Biology and Medicine, vol. 46, no. 5, pp. 531-542, 2009.

[10] A. M. Lefer and D. J. Lefer, "The role of nitric oxide and cell adhesion molecules on the microcirculation in ischaemiareperfusion," Cardiovascular Research, vol. 32, no. 4, pp. 743751, 1996.

[11] J. S. Kim, S. Ohshima, P. Pediaditakis, and J. J. Lemasters, "Nitric oxide: a signaling molecule against mitochondrial permeability transition- and $\mathrm{pH}$-dependent cell death after reperfusion," Free Radical Biology and Medicine, vol. 37, no. 12, pp. 1943-1950, 2004.

[12] G. Schinella, J. C. Fantinelli, and S. M. Mosca, "Cardioprotective effects of Ilex paraguariensis extract: evidence for a nitric oxidedependent mechanism," Clinical Nutrition, vol. 24, no. 3, pp. 360-366, 2005.

[13] L. M. Hung, M. J. Su, and J. K. Chen, "Resveratrol protects myocardial ischemia-reperfusion injury through both NO-dependent and NO-independent mechanisms," Free Radical in Biololgy and Medicine, vol. 36, no. 6, pp. 774-781, 2004.

[14] B. Haliwell, "Are polyphenols anti-oxidants or pro-oxidants? What do we learn from cell culture and in vivo studies?" Archives of Biochemistry and Biophysics, vol. 476, no. 2, pp. 107$112,2008$. 
[15] S. M. Mosca, G. R. Schinella, H. A. Tournier, and H. E. Cingolani, "Is the protection against ischemia induced by red wine linked to its antioxidant capacity?" Experimental and Clinical Cardiology, vol. 6, no. 4, pp. 183-187, 2001.

[16] J. C. Fantinelli, G. Schinella, H. E. Cingolani, and S. M. Mosca, "Effects of different fractions of a red wine non-alcoholic extract on ischemia-reperfusion injury," Life Sciences, vol. 76, no. 23, pp. 2721-2733, 2005.

[17] G. Schinella, J. C. Fantinelli, H. Tournier et al., "Antioxidant and cardioprotective effects of Ilex brasiliensis: a comparative study with Ilex paraguariensis (yerba mate)," Food Research International, vol. 42, no. 10, pp. 1403-1409, 2009.

[18] S. Yanagi, K. Matsumura, A. Marui et al., "Oral pretreatment with a green tea polyphenol for cardioprotection against ischemia-reperfusion injury in an isolated rat heart model," Journal of Thoracic and Cardiovascular Surgery, vol. 141, no. 2, pp. 511517, 2011.

[19] L. Djoussé, P. N. Hopkins, K. E. North, J. S. Pankow, D. K. Arnett, and R. C. Ellison, "Chocolate consumption is inversely associated with prevalent coronary heart disease: the national heart, lung, and blood institute family heart study," Clinical Nutrition, vol. 30, no. 2, pp. 182-187, 2011.

[20] H. G. Ávila, J. A. Cuspoca, G. Fischer, G. A. Ligarreto, and M. C. Quicazán, “Caracterización fisicoquímica y organoléptica del fruto de agraz (Vaccinium meridionale Swartz) almacenado a $2{ }^{\circ}$ C" Revista Facultad Nacional de Agronomía, Medellín, vol. 60, no. 2, pp. 4179-4193, 2007.

[21] G. A. Ligarreto, M. del Patiño, and S. V. Magnitskiy, "Phenotypic plasticity of Vaccinium meridionale (Ericaceae) in wild populations of mountain forests in Colombia," Revista de Biologia Tropical, vol. 59, no. 2, pp. 569-583, 2011.

[22] R. L. Prior, G. Cao, R. L. Prior, and G. Cao, "Analysis of botanicals and dietary supplements for antioxidant capacity: a review," Journal of AOAC International, vol. 83, no. 4, pp. 950-956, 2000.

[23] G. A. Garzón, C. E. Narváez, K. M. Riedl, and S. J. Schwartz, "Chemical composition, anthocyanins, non-anthocyanin phenolics and antioxidant activity of wild bilberry (Vaccinium meridionale Swartz) from Colombia," Food Chemistry, vol. 122, no. 4, pp. 980-986, 2010.

[24] A. Rommel, R. E. Wrolstad, and D. A. Heatherbell, "Blackberry juice and wine: processing and storage effects on anthocyanin composition, color and appearance," Journal of Food Science, vol. 57, no. 2, pp. 385-391, 1992.

[25] N. P. Seeram, Y. Zhang, and M. G. Nair, "Inhibition of proliferation of human cancer cells and cyclooxygenase enzymes by anthocyanidins and catechins," Nutrition and Cancer, vol. 46, no. 1, pp. 101-106, 2003.

[26] N. P. Seeram, "Recent trends and advances in berry health benefits research," Journal of Agricultural and Food Chemistry, vol. 58, no. 7, pp. 3869-3870, 2010.

[27] I. Ahmet, E. Spangler, B. Shukitt-Hale, J. A. Joseph, D. K. Ingram, and M. Talan, "Survival and cardioprotective benefits of long-term blueberry enriched diet in dilated cardiomyopathy following myocardial infarction in rats," PLoS ONE, vol. 4, no. 11, Article ID e7975, 2009.

[28] V. I. Singleton and J. A. Rossi Jr., "Colorimetry of total phenolics with phosphomolybdic acid reagents," American Journal of Enology and Viticulture, vol. 16, no. 3, pp. 144-158, 1965.

[29] C. Chiriboga and F. J. Francis, "An anthocyanin recovery system from cranberry pomace," Proceeding of the American Society of Horticultural Sciences, vol. 95, no. 2, pp. 233-236, 1970.
[30] A. Cavin, K. Hostettmann, W. Dyatmyko, and O. Potterat, "Antioxidant and lipophilic constituents of Tinospora crispa," Planta Medica, vol. 64, no. 5, pp. 393-396, 1998.

[31] R. Re, N. Pellegrini, A. Proteggente, A. Pannala, M. Yang, and C. Rice-Evans, "Antioxidant activity applying an improved ABTS radical cation decolorization assay," Free Radical Biology and Medicine, vol. 26, no. 9-10, pp. 1231-1237, 1999.

[32] I. F. F. Benzie and J. J. Strain, "The ferric reducing ability of plasma (FRAP) as a measure of "antioxidant power": the FRAP assay," Analytical Biochemistry, vol. 239, no. 1, pp. 70-76, 1996.

[33] W. H. Koppenol, R. Kissner, and J. S. Beckman, "Syntheses of peroxynitrite: to go with the flow or on solid grounds?" Methods in Enzymology, vol. 269, pp. 296-302, 1996.

[34] G. G. A. Balavoine and Y. V. Geletii, "Peroxynitrite scavenging by different antioxidants-part I: convenient assay," Nitric Oxide, vol. 3, no. 1, pp. 40-54, 1999.

[35] K. Jiao, S. Mandapati, P. L. Skipper, S. R. Tannenbaum, and J. S. Wishnok, "Site-selective nitration of tyrosine in human serum albumin by peroxynitrite," Analytical Biochemistry, vol. 293, no. 1, pp. 43-52, 2001.

[36] J. A. Buege and S. D. Aust, "Microsomal lipid peroxidation," Method in Enzymology, vol. 52, pp. 302-309, 1978.

[37] J. Sedlak and R. H. Lindsay, "Estimation of total, proteinbound, and nonprotein sulfhydryl groups in tissue with Ellman's reagent," Analytical Biochemistry, vol. 25, no. 1, pp. 192-205, 1968.

[38] J. M. Hare, "Nitroso-redox balance in the cardiovascular system," The New England Journal of Medicine, vol. 351, no. 20, pp. 2112-2114, 2004.

[39] N. Paolocc, U. E. G. Ekelund, T. Isoda et al., "cGMP-independent inotropic effects of nitric oxide and peroxynitrite donors: potential role for nitrosylation," American Journal of Physiology, vol. 279, no. 4, pp. H1982-H1988, 2000.

[40] F. J. Giordano, "Oxygen, oxidative stress, hypoxia, and heart failure," Journal of Clinical Investigation, vol. 115, no. 3, pp. 500508, 2005.

[41] P. Wang and J. L. Zweier, "Measurement of nitric oxide and peroxynitrite generation in the postischemic heart: evidence for peroxynitrite-mediated reperfusion injury," The Journal of Biological Chemistry, vol. 271, no. 46, pp. 29223-29230, 1996.

[42] T. Katori, S. Donzelli, C. G. Tocchetti et al., "Peroxynitrite and myocardial contractility: in vivo versus in vitro effects," Free Radical Biology and Medicine, vol. 41, no. 10, pp. 1606-1618, 2006.

[43] R. Bolli, "Causative role of oxyradicals in myocardial stunning: a proven hypothesis. A brief review of the evidence demonstrating a major role of reactive oxygen species in several forms of postischemic dysfunction," Basic Research in Cardiology, vol. 93, no. 3, pp. 156-162, 1998.

[44] B. Tavazzi, D. Di Pierro, M. Bartolini et al., "Lipid peroxidation, tissue necrosis, and metabolic and mechanical recovery of isolated reperfused rat heart as a function of increasing ischemia," Free Radical Research, vol. 28, no. 1, pp. 25-37, 1998.

[45] B. J. Michell, J. E. Griffiths, K. I. Mitchelhill et al., "The Akt kinase signals directly to endothelial nitric oxide synthase," Current Biology, vol. 9, no. 15, pp. 845-848, 1999.

[46] O. D. Saugstad, "Does nitric oxide prevent oxidative mediated lung injury?” Acta Paediatrica, vol. 89, no. 8, pp. 905-907, 2000.

[47] R. M. Clancy, D. Levartovsky, J. Leszczynska-Piziak, J. Yegudin, and S. B. Abramson, "Nitric oxide reacts with intracellular glutathione and activates the hexose monophosphate shunt 
in human neutrophils: evidence for S-nitrosoglutathione as a bioactive intermediary," Proceedings of the National Academy of Sciences of the United States of America, vol. 91, no. 9, pp. 36803684, 1994.

[48] P. C. Wootton-Beard and L. Ryan, "Improving public health? The role of anti-oxidant-rich fruit and vegetable beverages," Food Research International, vol. 44, no. 10, pp. 3135-3148, 2011. 


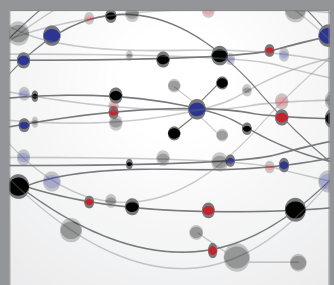

The Scientific World Journal
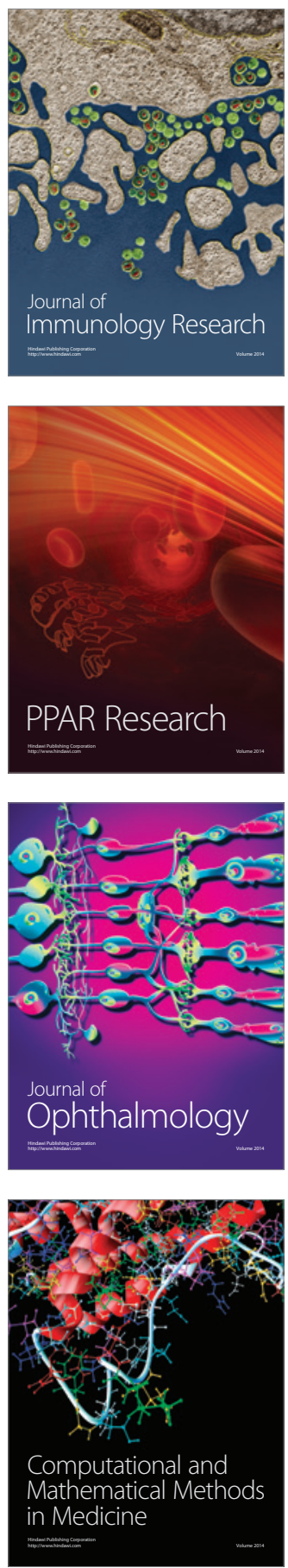

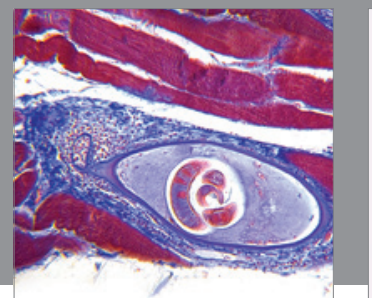

Gastroenterology

Research and Practice
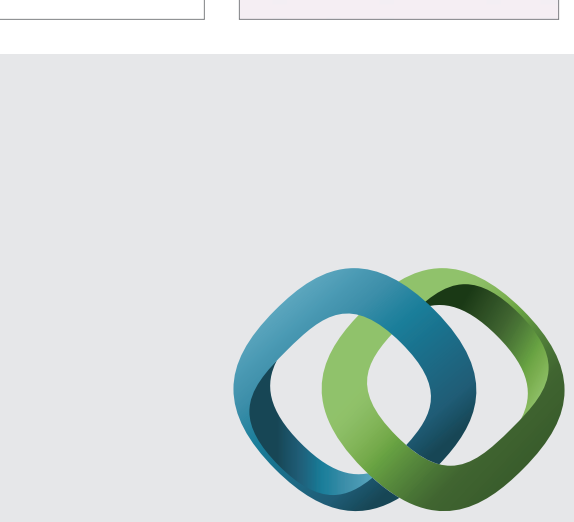

\section{Hindawi}

Submit your manuscripts at

http://www.hindawi.com
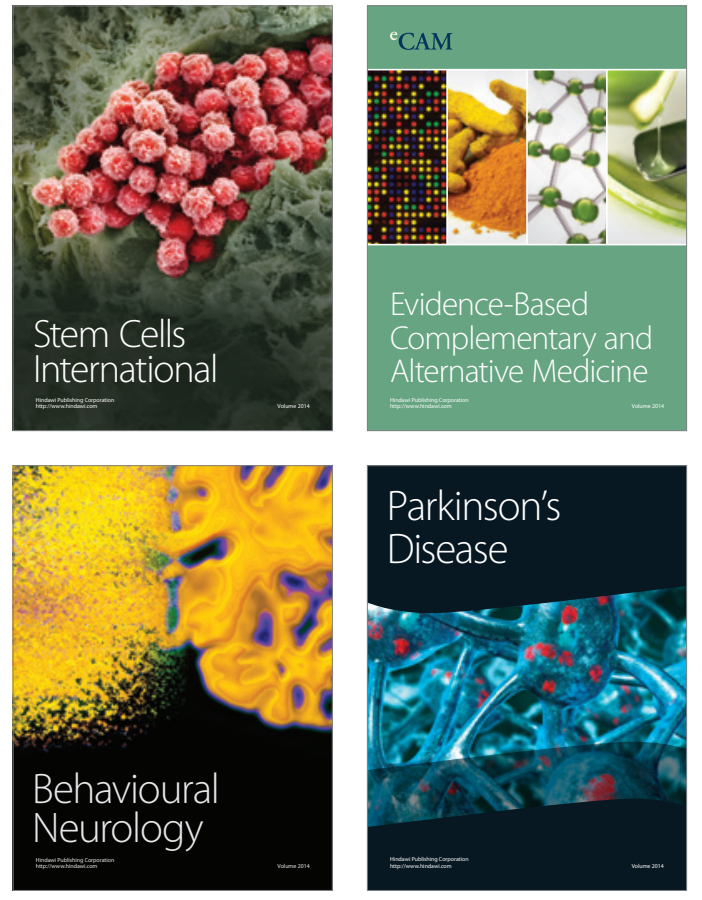
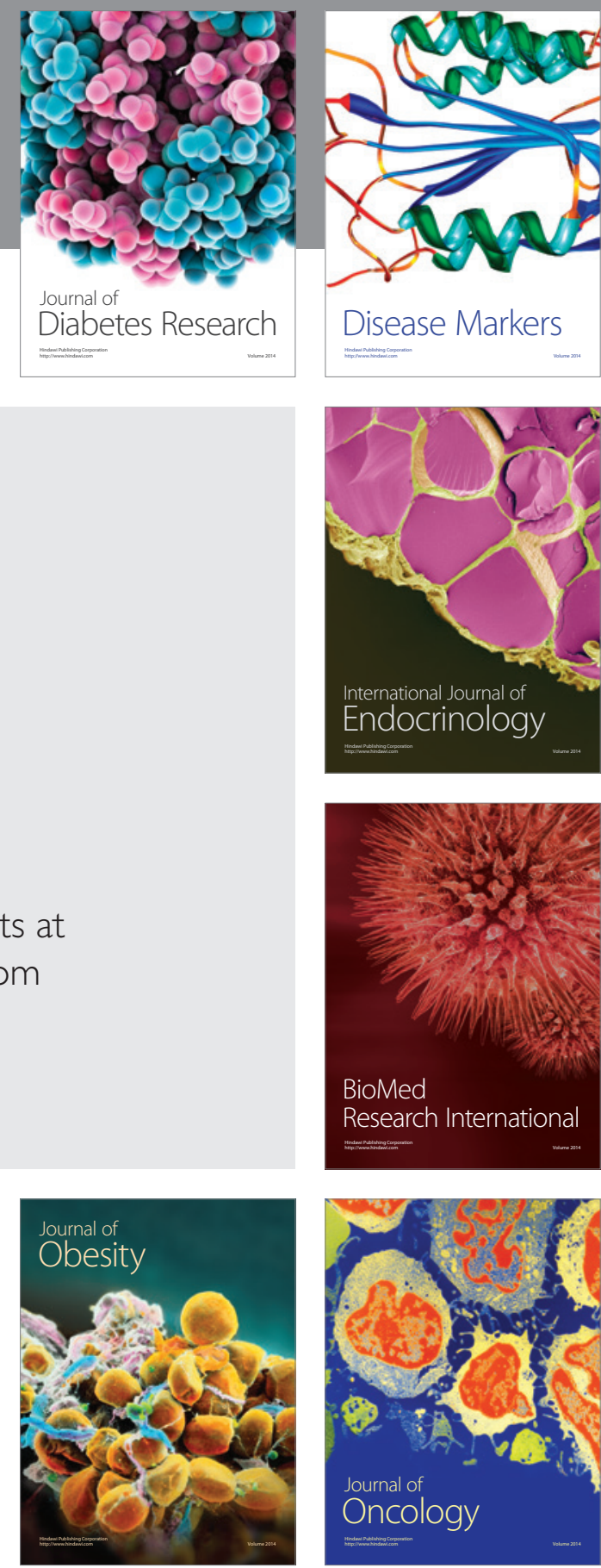

Disease Markers
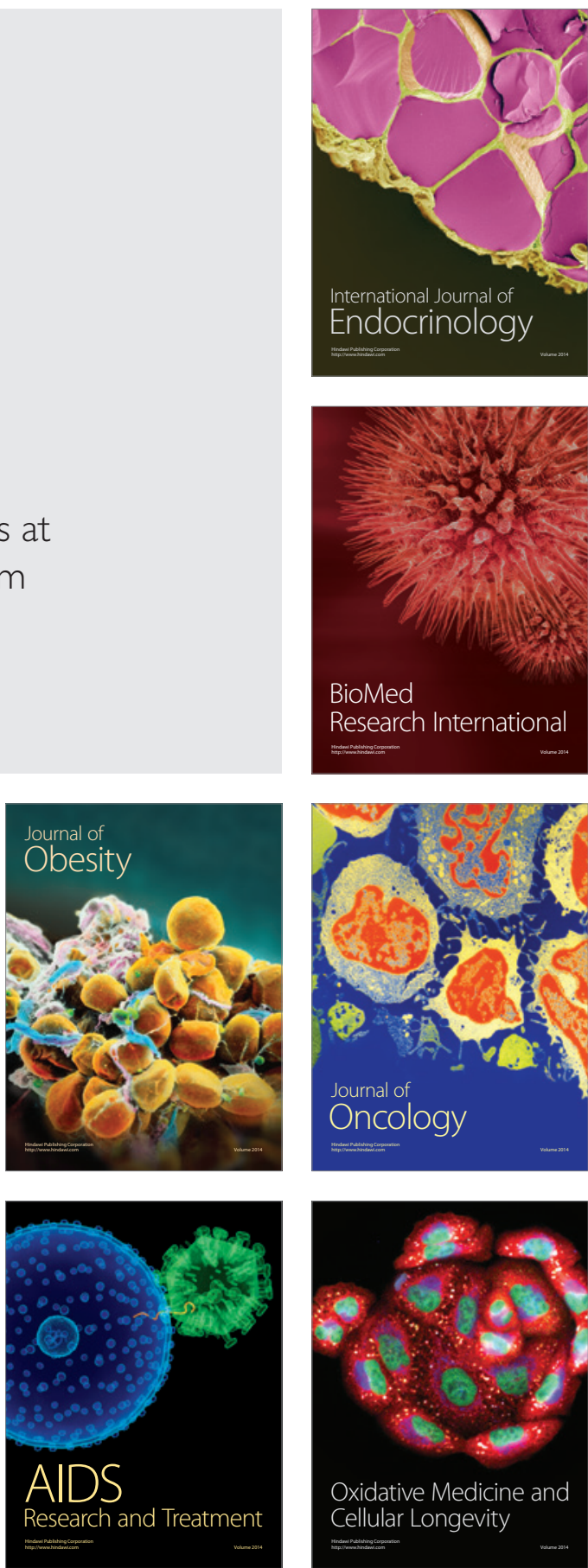